\title{
The Structure of Personality Capabilities
}

\author{
Delroy L. Paulhus and Carol Lynn Martin \\ University of British Columbia
}

\begin{abstract}
Wallace (1966) proposed that personality be construed as a set of abilities. Rather than assessing typical performance, as in trait ratings, he recommended assessing an individual's ability to perform social behaviors. We have elaborated this notion by distinguishing between personality abilities and capabilities. In this article we focus on the capability, that is, the ease with which an individual can display a certain category of social responses. A capability $X$ is assessed with self-reports of (a) likelihood of performing $X$ when perceived to be required, (b) perceived difficulty in performing $X$, (c) anxiety in performing $X$, and (d) tendency to avoid performing $X$. In Study 1 we examined the relations among six measures of 16 interpersonal behaviors in the context of the interpersonal circumplex. The four capability-related measures were shown to be measuring something distinct from the two trait measures. Unlike trait measures, which showed a circular structure in two dimensions, capability measures exhibited a positive manifold structure (i.e., no negative intercorrelations). The first two orthogonal factors were interpreted as Hostility and Nurturance, which are normally bipolar opposites on trait measures. Thus individuals capable of hostility are also capable of nurturance. The only dimension to remain bipolar was introversion-extraversion. In Study 2 , the nomological network of the capability measures was shown to be consistent with the theoretical construct. For example, high self-esteem and interpersonal control were associated with almost all of the interpersonal capabilities.
\end{abstract}

Almost 20 years ago, Wallace $(1966,1967)$ elaborated the view that personality attributes are better construed as abilities than as traits. He noted that an individual's tendency to display a behavior is a function of the ability to perform the behavior moderated by situational variables like inhibition and lack of incentive. Thus individuals may not typically be dominant either because they are not able to be dominant (i.e., they lack the necessary skills) or because their dominant predisposition is typically inhibited. By failing to address this distinction, standard trait conceptions of typical or average behavior confound ability deficits with response inhibition. To assess a person's response ability, Wallace argued that the assessor must provide the optimal situation for that person to display it. Once such an ability is confirmed (or disconfirmed), the assessor is in a better position to make a decision regarding job placement, social skills training, or psychotherapy, as the case may be.

The ability approach to personality has been largely ignored by personologists. One exception was Fiske (1971), who accorded ability measurement the status of one of the six primary modes of personality assessment. In fact, in an earlier article, Fiske and Butler (1963) anticipated the ability approach. More recently, Fishbein and Ajzen (1975) argued that a limited capability is one of few factors that can interfere with the strong relation between intention and performance of behavior. Nevertheless, none of these writers has conducted any empirical work on personality abilities.

We wish to thank Lynn Alden, Ross Broughton, Bill Graziano, Lew Goldberg, Oliver John, and Jerry Wiggins for comments on an earlier draft.

Correspondence concerning this article should be addressed to Delroy L. Paulhus, Department of Psychology, University of British Columbia, Vancouver, British Columbia, Canada V6T IW5.
It was some time later that Willerman, Turner, and their colleagues developed a useful operationalization (Klein \& Willerman, 1979; Turner, 1978; Willerman, Turner, \& Peterson, 1976). Following Wallace and Fiske, they argued that abilities should be assessed by measuring the individual's maximal performance. They administered both typical and maximal measures of personality: Typical measures assess traits, that is, average or most frequent behavior; ${ }^{1}$ maximal measures assess the most extreme behavior of which the person is capable. On selfreport instruments, the format for typical measures is, for example, "How aggressive are you?" whereas the format for maximal measures is "How aggressive are you capable of being?" For behavioral assessment in the laboratory, the role-playing instructions for typical measures are "Behave as aggressively as you typically would." For maximal measures, the instructions are "Behave as aggressively as you are capable of being" (Klein \& Willerman, 1979).

The properties of maximal measures have been examined in several studies. For example, maximal self-reports were shown to predict maximal behavior better than did typical self-reports (Turner, 1978; Willerman et al., 1976). In addition, maximal self-reports predicted maximal peer ratings better than typical self-reports predicted typical peer ratings (Klesges \& McGinley, 1983). Surprisingly, in the Turner (1978) study, the maximal

\footnotetext{
${ }^{1}$ Following Willerman, Turner, and associates' terminology, we will use the term trait in its phenotypic (surface trait) sense as a summary of observed behavior (D. M. Buss \& Craik, 1980; Cattell, 1957; McClelland, 1951; Wiggins, 1974). This usage is appropriate for this investigation, where respondents make global ratings of their typical behavior. Of course, the term trait is often used in its genotypic (source trait) sense, referring to the underlying structure responsible for observed consistency (Cattell, 1957; Hirschberg, 1978; Ryle, 1949). Wallace's $(1966,1967)$ critique of traits is directed at the genotypic sense.
} 
self-reports predicted typical laboratory behavior significantly better than did typical self-reports.

Maximal assessment has also proved useful in addressing a number of substantive issues. For example, Klein and Willerman (1979) found that under typical instructions, college women were less dominant with a male confederate than with a female confederate. Under maximal instructions ("be as dominant as you can"), however, the women acted equally dominant with confederates of both sexes. Because women's dominance increased under maximal instructions, the authors concluded that women normally inhibit dominance in interactions with men. Similarly, Turner (1983) demonstrated that socially anxious students were rated less dominant than nonanxious students under typical instructions but not under maximal instructions. Turner concluded that social anxiety is best interpreted as a problem of inhibition rather than a social skills deficit (see also Nietzel \& Bernstein, 1976; Wolpe, 1969).

The ability approach outlined by Wallace has been useful for distinguishing between the ability to perform behaviors and the actual performance of these behaviors. An ability may fail to be demonstrated for a variety of reasons: lack of opportunity, lack of motivation, anxiety, or situational constraints. Some factors, however, may be easier than others to separate from the underlying ability. We will argue that in making self-reports, the individual's perception of his or her response ability is not separable from the anxiety associated with performing the response.

\section{Varieties of Personality Abilities}

The argument presented in this article is based on distinguishing between response ability and response capability, terms that have been used interchangeably by other writers. We will use the term personality ability to mean the degree of skill with which an individual can execute a particular social routine under optimal conditions. An ability includes knowing what to do and how to do it. If deficits in such abilities are serious, the individual may have to be taught how to execute new responses (Hersen \& Eisler, 1976; Twentyman \& McFall, 1975; White \& Berger, 1976). For example, socially unskilled patients who were trained in socially skilled behavior subsequently experienced less anxiety (Percell, Berwick, \& Beigel, 1974) and were viewed as more socially attractive (Lewinsohn, Weinstein, \& Alper, 1970). Abilities are best measured in maximal situations (Wallace, 1966); that is, given the optimal circumstances, can the behavior be performed? One such approach involves assessing maximal traits, as in the work of Willerman, Turner, and their associates: Respondents are asked to report the most extreme level of behavior of which they are capable. The rationale is that the more able person can perform the more extreme behaviors on that dimension. ${ }^{2}$

In contrast, we use the term personality capability to refer to the ease of carrying off a particular response when required by the situation. Thus, a capability assessment must capture the ability to perform a response modulated by the conditioned emotional response (primarily anxiety) associated with performing the behavior. Anxiety will not only lower the probability of attempting the behavior but will also disrupt the performance if it is attempted. Capabilities exclude other motivational factors. This applies to both intrinsic motivation (e.g., need for approval), which may energize the response, and exter- nal motivation (e.g., incentives), which increases the probability of a response. ${ }^{3}$

The capability may be a more useful unit of analysis than the raw ability to perform a social behavior. Although it is possible conceptually to distinguish abilities and anxiety, they are closely associated in actual behavior (Bandura, 1982). Indeed, anxiety level appears to be an inextricable component of social performance in a reciprocal loop of cause and effect (Leary, 1957). In the incapable individual, the mere anticipation of performance seems to trigger an anxious reaction. Of course, low ability necessarily begets low capability. However, the large majority of normal individuals seem to possess the basic social knowledge and performance skills. Rather, it is individual differences in social anxiety that explain more of the variation across people (Arkowitz, Lichenstein, McGovern, \& Hines, 1975). Most important, when making self-reports, respondents are unlikely to be able to distinguish these two sources of incapability.

In this investigation, capabilities will be assessed by self-reported ease and likelihood of performance when a certain response is called for by the situation. ${ }^{4}$ Individuals are considered capable of dominance, for example, if they can be dominant when they feel it is required. Usually the requirement is to be socially appropriate. Because appropriateness is defined in subjective terms, capability measures index, in effect, the congruence between the respondent's real and ideal behavior. Although subjective, these judgments are hardly idiosyncratic, given the strong consensus in people's judgments of what responses are socially appropriate (Edwards, 1957). Finally, the subjective definition of what response is appropriate allows for alternative, but equally effective, responses to the same situation.

In sum, we propose a distinction among three psychometric levels: Ability measures assess degree of skill, capability measures assess the ease of displaying the appropriate response, and trait measures index typical behavior. We suggest that all three levels are necessary to understand the structure of personality.

\section{Structure of Traits and Abilities}

One of the best established results in personality assessment is the circumplex structure of interpersonal traits (Leary, 1957; Wiggins, 1979). Specifically, the set of 16 traits anchored by the dominance/submission and nurturance/hostility axes invariably exhibits a circular pattern under factor analysis. A comparable circumplex emerges in analyses of affect (Russell, 1980) and motives (Smith, 1984; Wiggins \& Broughton, 1985). The robustness of the circumplex appears to derive from its basis in the two fundamentals of interpersonal exchange, namely love and status (Foa \& Foa, 1974; Sullivan, 1953).

\footnotetext{
${ }^{2}$ Extreme behavior may be the culmination of prolonged overcontrol rather than an indication of a strong trait (Megargee, 1966).

${ }^{3}$ Self-reported traits are a summary of recent behavior, which is affected by motivation and recent situational presses as well as capabilities. Situational presses include the individual's current reward and punishment structure as well as the degree of freedom to self-select into preferred situations (Gormly, 1983). We would expect, therefore, that self-reported capabilities should be more stable than traits (typical behavior).

${ }^{4}$ The capabilities dealt with in this article are all functional, that is, appropriate for the situation. Dysfunctional capabilities will be addressed in another article.
} 
Although Wallace recommended some time ago (Wallace, 1967) that the structure of personality abilities be investigated, virtually no research has addressed the issue. To date, the only known study is an investigation of the structure of maximal traits (Broughton \& Paulhus, 1984). For each of the 16 trait terms from the circumplex, subjects gave self-ratings on maximal behaviors (e.g., "How dominant are you capable of being?"). A factor analysis of the intercorrelations did not yield the circumplex structure found with standard trait ratings. Rather, the maximal traits showed no negative intercorrelations, instead forming a positive manifold.

The results from the Broughton and Paulhus (1984) study suggest that the structure of ability-related measures may be distinct from the structure of standard trait ratings. In light of these findings, we expect that a structural analysis of interpersonal capabilities will also differ from the standard trait structure. Similar to maximal traits, a capability for one interpersonal behavior should not constrain in any systematic way the possession of other capabilities. For example, individuals may be capable of both dominance and submissiveness. Hence, we expect a converging pattern similar to that found by Broughton and Paulhus (1984), that is, a positive manifold pattern rather than a circumplex.

\section{Present Investigation}

The first goal of the studies presented here was to develop a self-report instrument to assess a wide variety of interpersonal capabilities. The 16 traits of the interpersonal circumplex (Wiggins, 1979) were used as the domain of attributes. Given that the circumplex includes a full range of positive as well as negative attributes, it seems a better choice than the Moe and Zeiss (1982) battery, which includes only positive attributes. The second goal was to examine the psychometric properties of interpersonal capabilities and compare them to standard trait ratings. The third goal was to investigate the structural relations among the 16 interpersonal capabilities. On the basis of the results of the Broughton and Paulhus( 1984) study, we predict that the circumplex structure will shift toward a positive manifold structure. The fourth goal was to assess the convergent and discriminant validity of the capability construct in relation to a number of standard personality measures.

\section{The BIC Inventory}

A self-report inventory was developed to assess functional capabilities in the interpersonal sphere: the Battery of Interpersonal Capabilities (BIC). Respondents are asked four questions about their capability of enacting each of 16 interpersonal behaviors in appropriate situations. For each behavior, subjects are asked a global capability question, for example, "How likely is it that you could be dominant if the situation requires it?" This question is the most direct index because it addresses manifest behavior. To assess discomfort, respondents are asked to rate (a) the difficulty of performing each behavior, (b) anxiety when performing each behavior, and (c) the tendency to avoid situations demanding such behavior. Responses to all questions were rated on 7-point Likert scales anchored by very much (7) and not at all (1).

\section{Study 1}

\section{Method}

Subjects. Respondents were from five undergraduate psychology courses at a large Canadian university. A total of 252 respondents ( 102 men, 150 women) completed several questionnaires anonymously in large groups.

Procedure. Subjects were given the BIC and two standard trait-rating questionnaires. Thus, subjects provided self-ratings on the 16 traits from the interpersonal circumplex in a total of six different formats of assessment. We have already described the four question formats of the BIC; the fifth format was a standard trait rating. All ratings were made on 7-point Likert scales anchored by very much (7) and not at all (1). Finally, the same 16 traits were assessed by using a three-level variation of Goldberg's (1981) six-choice trait-rating scheme: $1=$ not accurate as a description; 2 = average or middle; $3=$ accurate as a description. Also scored as a 2 were two other response choices: It depends on the situation and I'm uncertain. Respondents choosing I don't understand the term (a total of 5) were dropped from all analyses.

\section{Results}

To simplify our presentation, we will use the term capability to refer to the likelihood item on the BIC. Table 1 displays the mean rating for each question mode on each of the 16 traits. Note first that every capability rating is higher than the corresponding trait rating. This result is consistent with the assumption that individuals are capable of higher levels of interpersonal behaviors than they typically display. Note second that respondents generally report the most difficulty in performing antisocial behaviors, that is, those on the left side of the circumplex (cold, quarrelsome, arrogant, etc.). These negative behaviors are given lower ratings on the capability mode and higher ratings on the three discomfort modes, difficulty, anxiety, and avoidance.

Structural relations among modes. For each of the 16 attributes, correlations were calculated among six measures of that attribute: the four BIC modes plus the standard trait rating and the Goldberg trait rating. All $166 \times 6$ correlation matrices were similar in pattern. The overall $6 \times 6$ correlation matrix (Table 2 ) was assembled by averaging across the 16 traits. To calculate each entry in this matrix, Fisher's $r$-to- $z$ transformations were used to average the 16 corresponding correlations.

This correlation matrix was subjected to principal-factor analysis followed by varimax rotation. Only the first two factors had eigenvalues above unity, and together they accounted for $63 \%$ of the total variance. The factor loadings of the six assessment modes are displayed in Figure 1. The factor loadings on the three discomfort modes were reversed for display purposes to clarify the clusters. As seen in the figure, the standard trait ratings and the Goldberg ratings load highly on one factor, whereas the capability, difficulty, anxiety, and avoidance ratings load on the other factor. ${ }^{5}$ The emergence of two clusters from the factor analysis makes one point clear: The four capability-

\footnotetext{
${ }^{5}$ To rule out acquiescence and extremity artifacts, we refactored the correlations after partialing out subjects' extremity scores on the trait ratings. The pattern was virtually unchanged.
} 
related measures are tapping a construct different from that assessed by standard trait measures of personality.

Structural relations among traits and capabilities. The correlations among the 16 attributes were calculated separately for each of the six modes. A principal-factor analysis with varimax rotation was performed on each of the six correlation matrices.

As recommended by Wiggins (1980), adjacent pairs of traits were combined before factoring. The structure of the standard trait ratings is evident from the factor loadings plotted in Figure 2. This typical circular ordering has been replicated many times before (see Wiggins, 1980). A similar pattern emerged using Goldberg's ratings. Note that all previous circumplex studies have used composite indexes for each trait. Our data demonstrate that the circumplex structure can also be obtained using global trait ratings (see Burisch, 1984). (Unlike in previous studies, however, we anchored each trait firmly by providing several synonyms.)

In contrast, the factor structure for the general capability mode shows the distinctive positive manifold pattern seen in Figure 3. In fact, all four capability question modes showed similar patterns. The interpersonal capabilities all collapsed into the first quadrant. For all four capability-related modes, the same two major factors emerged. One factor, marked by warm and agreeable, was labeled Nurturance. The second factor, marked by cold and quarrelsome, was labeled Hostility. Recall that for trait ratings, these two clusters mark the opposite poles of the major horizontal axis of the circumplex. The third orthogonal factor was marked at opposite poles by extraversion and introversion.

\section{Discussion}

The first structural analysis explored the relations among the four capability-related measures on the BIC and two forms of

Table 1

Item Means Across Modes and Attributes

\begin{tabular}{lccccc}
\hline & \multicolumn{5}{c}{ Question mode } \\
\cline { 2 - 6 } \multicolumn{1}{c}{ Attribute } & Capability & Low & Low & Low \\
difficulty & anxiety & avoidance & Trait \\
\hline Dominant & 5.64 & 4.77 & 4.43 & 4.70 & 4.42 \\
Ambitious & 6.20 & 5.26 & 4.58 & 4.22 & 5.52 \\
Extraverted & 5.59 & 4.62 & 4.46 & 4.84 & 4.62 \\
Gregarious & 6.06 & 5.27 & 4.96 & 5.16 & 4.99 \\
Agreeable & 6.15 & 5.57 & 5.40 & 5.51 & 5.12 \\
Warm & 6.24 & 5.56 & 5.51 & 5.61 & 5.48 \\
Trusting & 5.50 & 5.01 & 4.23 & 4.99 & 3.09 \\
Unassuming & 5.78 & 5.34 & 5.45 & 5.32 & 4.19 \\
Submissive & 4.53 & 3.95 & 3.79 & 3.59 & 3.01 \\
Lazy & 5.23 & 5.13 & 4.98 & 4.74 & 3.53 \\
Introverted & 5.10 & 4.77 & 4.70 & 4.53 & 3.22 \\
Aloof & 5.09 & 4.58 & 4.44 & 4.19 & 3.29 \\
Quarrelsome & 4.69 & 3.93 & 3.45 & 3.02 & 3.19 \\
Cold & 4.52 & 3.84 & 3.66 & 3.24 & 2.15 \\
Calculating & 5.30 & 4.69 & 4.21 & 4.19 & 4.10 \\
Arrogant & 4.33 & 3.73 & 3.51 & 3.30 & 2.69 \\
$\quad$ Mode & & & & & \\
$\quad$ mean & 5.37 & 4.75 & 4.46 & 4.45 & 3.91 \\
\hline
\end{tabular}

Note. Self-ratings are all on 7-point Likert scales. Values in the table are unweighted means across 102 men and 150 women.
Table 2

Correlations Among Six Modes of Self-Ratings

\begin{tabular}{|c|c|c|c|c|c|c|}
\hline \multirow[b]{2}{*}{ Variable } & \multicolumn{4}{|c|}{ Capabilities } & \multicolumn{2}{|c|}{ Traits } \\
\hline & 1 & 2 & 3 & 4 & 5 & 6 \\
\hline \multicolumn{7}{|l|}{ Capabilities } \\
\hline 1. Global capability & - & & & & & \\
\hline 2. Low difficulty & .59 & - & & & & \\
\hline 3. Low anxiety & .46 & .60 & - & & & \\
\hline 4. Low avoidance & .45 & .52 & .53 & - & & \\
\hline \multicolumn{7}{|l|}{ Traits } \\
\hline 5. Standard & .31 & .30 & .28 & .29 & - & \\
\hline 6. Goldberg & .27 & .23 & .18 & .26 & .58 & - \\
\hline
\end{tabular}

Note. $N=252$. All correlations are significant under a two-tailed test, $p<.01$. This correlation matrix is the average of 16 similar matrices, one for each of the 16 circumplex traits.

trait ratings. Factor analyses showed a clustering of capability measures separate from the trait measures. This is an important preliminary demonstration that capability measures are tapping some common construct. Moreover, this underlying construct is psychometrically distinct from the more traditional trait concept.

This pattern of results is consistent with the theoretical relations among traits, capabilities, and inhibition as specified in the introduction. To begin with, global capabilities were not independent of inhibition measures, in particular, reported anxiety. Clearly, the anxiety anticipated in performing a social behavior plays a major role in an individual's judgment about his or her capability of performing the behavior. The data, however, do not allow us to unconfound the impact that anticipated anxiety has on the capability judgment from the impact that the capability judgment has on anticipated anxiety. Indeed, as we argued earlier, it may be best to leave them confounded in selfreported capabilities.

A second aspect of the structural analysis bears on our theoretical assumptions. Capability and trait assessments of the same behavior are not orthogonal but show moderate positive correlations in the .25 range. Therefore, capability judgments are not just wishful thinking, which would be implied by zero correlations with traits. Rather, this pattern is consistent with our earlier statements that a high capability enhances the probability of typically displaying the behavior but does not completely determine it.

The second structural analysis tested whether the usual circumplex structure of Wiggins's (1979) set of 16 interpersonal traits would be sustained for capability ratings. Although the circumplex was replicated for standard trait ratings, the capability ratings exhibited a dramatically different three-dimensional structure. Here, the usual circular ordering in two dimensions collapsed into the first quadrant. This novel structure was marked by two orthogonal clusters labeled Hostility and Nurturance. Recall from Figure 2 that in the trait structure, these two clusters are polar opposites.

This difference in patterns of trait and capability ratings has important implications for the nature of personality. Because trait measures require respondents to fix themselves at some point on the rating scale, a trait and its semantic opposite must be negatively correlated. It stands to reason that an individual 


\section{Relations Among Six Modes of Personality Assessment}

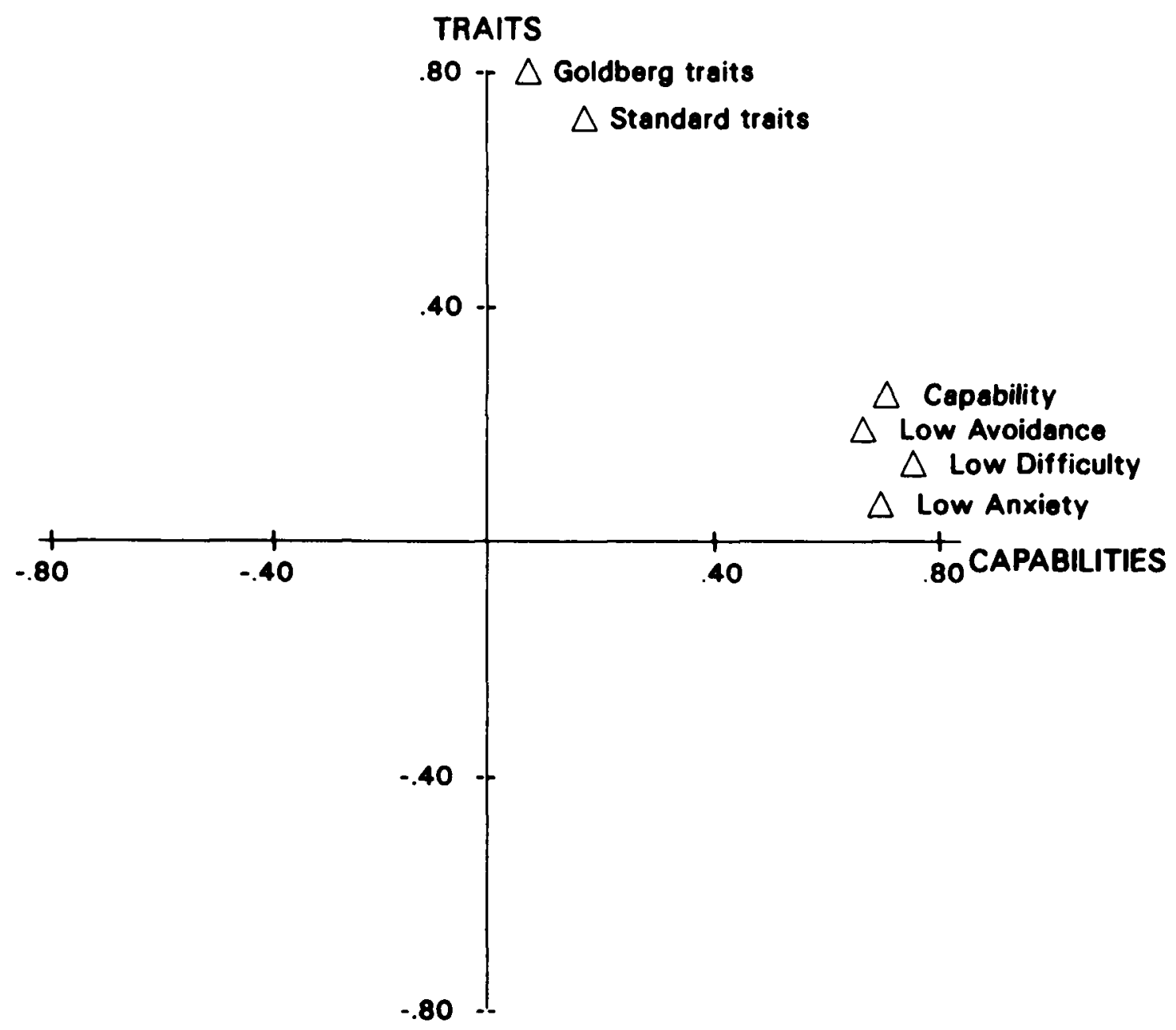

Figure 1. First two principal factors of correlations among modes in Table 2.

who is typically warm will not also report being typically cold. Our data, however, indicate that an individual who is capable of warm behavior will also, as likely as not, be capable of cold behavior. Moreover, the individual who is incapable of warm behavior is not necessarily capable of cold behavior. In fact, the positive manifold pattern indicates that individuals who report one capability tend to report other capabilities (see Footnote 5). The capabilities for dominance and extraversion, lying at the center of the manifold, may be fundamental to all other capabilities.

Finally, a third bipolar factor, introversion-extraversion, emerged from the factoring of trait capabilities. This pair of traits, which were bipolar in the standard circumplex structure, remained bipolar in the capability structure. Individuals rating themselves as capable of extraversion also rated themselves as incapable of introversion. Similarly, those capable of introversion were incapable of extraversion. This constraint on capabilities may be a biological one. Of all the traits composing the circumplex, introversion-extraversion has the strongest evidence for biological underpinnings (A. H. Buss \& Plomin, 1975; Eysenck \& Eysenck, 1969). In particular, this dimension shows the highest heritabilities of any personality dimension ${ }^{6}$ (Loehlin \& Nichols, 1976; Shields, 1976). Moreover, extraversion has been linked with minor physical anomalies of congenital origin (Paulhus \& Martin, 1986). To the extent that interpersonal traits are biological in origin, capabilities are necessarily constrained.

\section{Study 2}

One way of supporting the construct validity of the capability measures developed here is to show that they are coherently embedded in the related nomological network. This process includes (a) convergent validation, which is the demonstration of

\footnotetext{
${ }^{6}$ The sociability component of extraversion, not impulsivity, appears to carry most of the heritability (A. H. Buss \& Plomin, 1975).
} 


\section{The Structure of Standard Trait-Ratings}

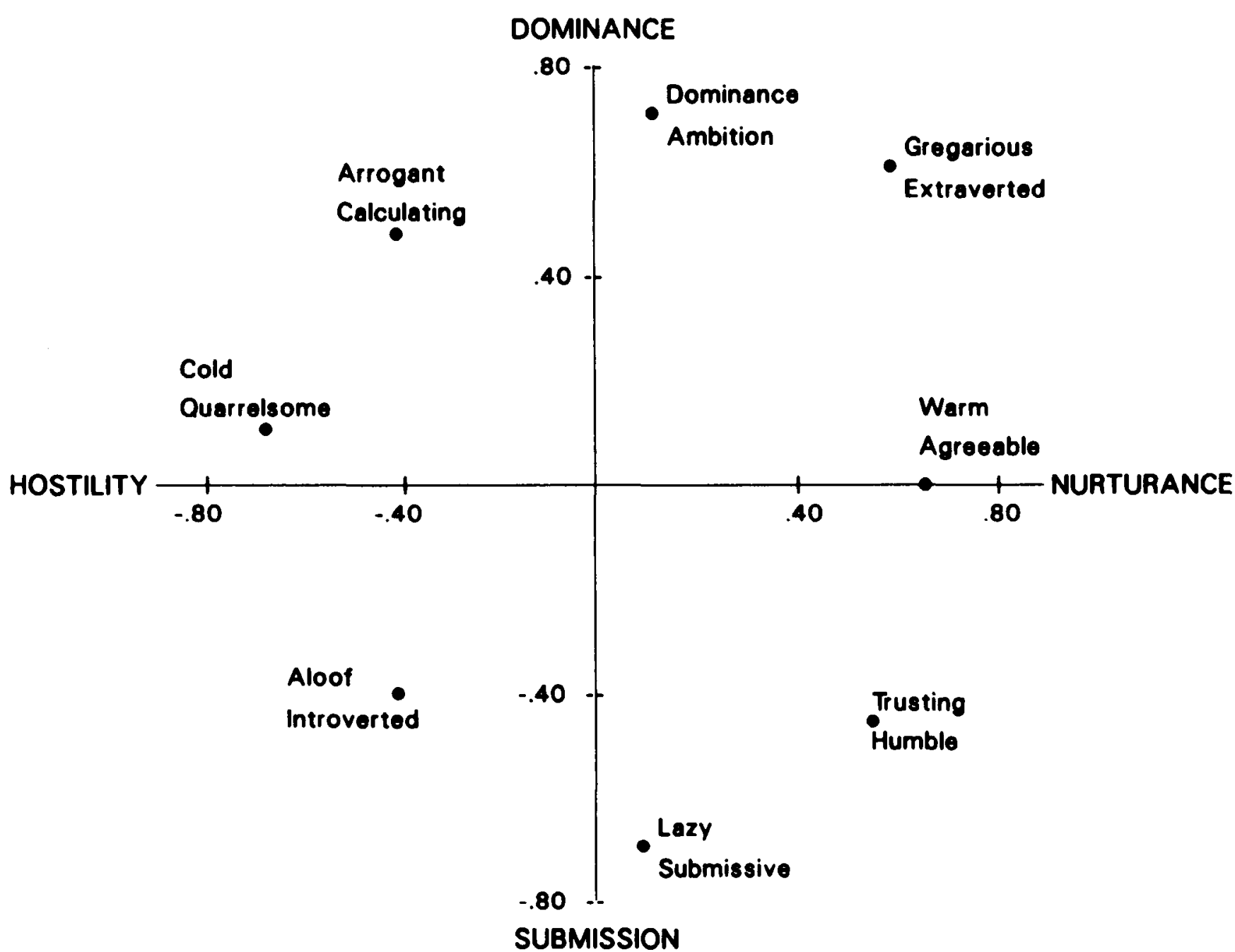

Figure 2. First two principal factors of standard trait-ratings.

predictable relations with conceptually related measures, and (b) discriminant validation, which is the demonstration of null relations with conceptually unrelated measures (Campbell \& Fiske, 1959).

For instance, one potential confound in asking people about their capabilities is socially desirable responding. Individuals with a set to respond in a socially desirable fashion may well claim to be capable of performing any interpersonal behavior. To investigate this possibility, an established measure of socially desirable responding should be administered along with the BIC. In this study, we used the Marlowe-Crowne Social Desirability Scale (Crowne \& Marlowe, 1960) because it is the most frequently used measure and it taps both forms of socially desirable responding: self-deception and impression management (Paulhus, 1984). ${ }^{7}$

Convergent validation for the capability measures would accrue from demonstrating relations with established measures of constructs related to interpersonal capabilities. One measure selected for this purpose was the Interpersonal Control scale from the Spheres of Control battery (Paulhus, 1983). This scale assesses perceived control over social interactions in groups and in dyads. The individual high in interpersonal control should be capable of a variety of positively oriented social behaviors like dominance and warmth. A second established measure relevant to interpersonal capabilities is the Machiavellianism scale, which assesses the tendency to manipulate others using duplicitous tactics (Christie \& Geis, 1970). The Machiavellian individual should be particularly capable of the more antisocial interpersonal behaviors in the circumplex, for example, hostility and coldness.

Many writers have postulated a close link between interper-

\footnotetext{
${ }^{7}$ Unfortunately, the two components cannot be assessed separately using the Marlowe-Crowne Social Desirability Scale. The problem is that the items tend to load simultaneously on both factors and therefore cannot be partitioned into separate clusters (Paulhus, 1984).
} 


\section{Structure of Personality Capabilities}

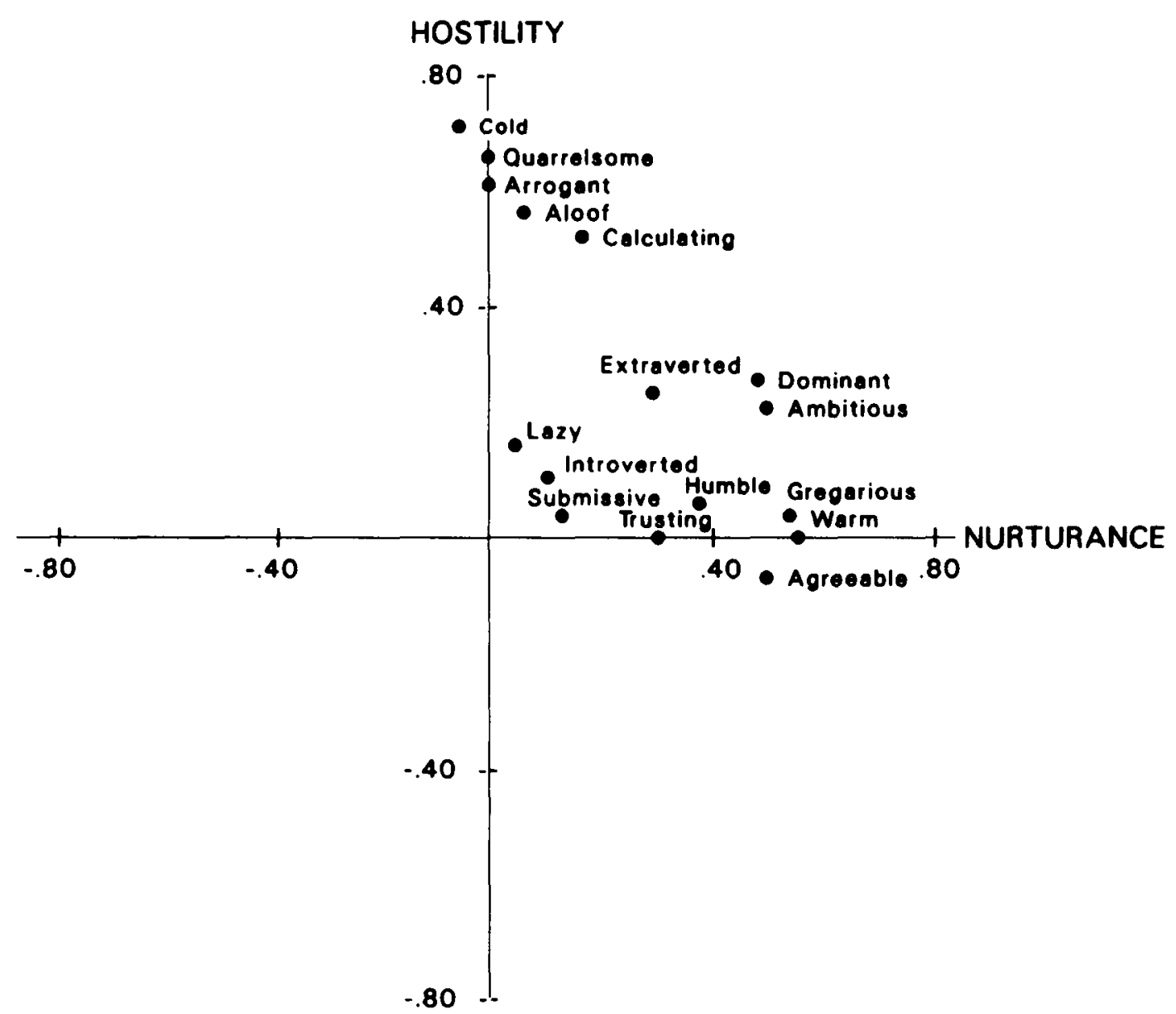

Figure 3. First two principal factors of personality capabilities.

sonal capabilities and adjustment (e.g., Leary, 1957; Sullivan, 1953). Leary in particular argued that individuals who can adjust their behavior to suit the situation should enjoy the maximum interpersonal success. Such success would then contribute to a sense of well-being by way of high self-esteem and low anxiety (Leary, 1957). Some of Leary's predictions were sustained in our recent research (Martin \& Paulhus, 1984; Paulhus $\&$ Martin, 1985). We found that functionally flexible individuals, that is, those who reported a wide range of interpersonal capabilities, also reported high self-esteem. We did not investigate whether some capabilities contributed more than others to self-esteem; rather we treated all capabilities as equivalent contributors to flexibility.

Accordingly, in Study 2 we examined the contribution of each of the 16 circumplex capabilities to several varieties of adjustment. Block's (1965) Ego-Resiliency Scale was inciuded because it is a widely used measure of the first factor of the Minnesota Multiphasic Personality Inventory (MMPI), a general adjustment factor. Also included were Taylor's (1953) Manifest Anxiety Scale and Rosenberg's (1965) Self-Esteem Scale, the most widely used measures of anxiety and self-esteem, respectively.

\section{Method}

A total of 164 undergraduates ( 79 men, 85 women) from two classes completed a battery of questionnaires in large group administrations. The battery included the BIC as well as the Marlowe-Crowne Social Desirability Scale, Paulhus's Interpersonal Control scale, the Rosenberg Self-Esteem Scale, Block's Ego-Resiliency Scale, the Mach IV scale, and the Manifest Anxiety Scale.

\section{Results}

For each circumplex behavior, the correlations between the general capability measure and the six personality and adjustment measures are presented in Table 3. The sample sizes differ across measures not because of selective returns but because undergraduate classes of varying sizes were given different combinations of measures.

Note first that the correlations between the Marlowe-Crowne Social Desirability Scale and the various capabilities are low; none reaches significance. In contrast, the Interpersonal Control scale exhibits significant positive correlations with 9 of the 16 interpersonal capabilities. As expected, the correlations are highest for the more positive, prosocial capabilities like domi- 
Table 3

Personality Correlates of Interpersonal Capabilities

\begin{tabular}{|c|c|c|c|c|c|c|}
\hline Attribute & \multicolumn{6}{|c|}{ Personality and adjustment measures } \\
\hline Dominant & $-.29^{* *}$ & $.25^{* *}$ & .12 & $.51^{* *}$ & .06 & -.16 \\
\hline Ambitious & $-.22^{*}$ & $.32^{* *}$ & $.20^{*}$ & $.49^{* *}$ & $.29^{*}$ & .08 \\
\hline Extraverted & $-.20^{*}$ & $.42^{* *}$ & $.28^{*}$ & $.57 * *$ & -.16 & .22 \\
\hline Gregarious & .03 & $.26^{* *}$ & .13 & $.48^{* *}$ & .15 & .01 \\
\hline Trusting & $.20^{*}$ & $.16^{*}$ & .11 & $.27^{*}$ & -.15 & .04 \\
\hline Humble & .00 & .14 & -.06 & -.02 & -.13 & -.13 \\
\hline Submissive & $.30^{* *}$ & -.15 & -.16 & -.18 & -.17 & -.03 \\
\hline Lazy & $.19^{*}$ & -.02 & -.13 & -.10 & -.09 & .07 \\
\hline Introverted & $.19^{*}$ & -.14 & -.16 & -.15 & .20 & -.24 \\
\hline Aloof & -.05 & .06 & -.03 & .10 & .17 & -.04 \\
\hline
\end{tabular}

Note. The sample sizes vary across measures because varying sizes of undergraduate classes completed different measures. Anxiety was assessed with Taylor's Manifest Anxiety Scale, self-esteem with Rosenberg's Self-Esteem Scale, ego resiliency with Block's Ego-Resiliency Scale, interpersonal control with Paulhus's Interpersonal Control scale, Machiavellianism with Christie's Mach IV scale, and social desirability with the MarloweCrowne Social Desirability Scale.

$p<.05$, two-tailed.

$* * p<.01$, two-tailed.

nance, ambition, extraversion, gregariousness, and warmth. A notable exception is agreeable, which unexpectedly shows only a small positive correlation. The correlations with Mach IV are, as predicted, highest for the antisocial capabilities like cold, calculating, and quarrelsome. In addition, the Mach IV showed a moderate relation with a capability for ambition.

The correlations with self-esteem are in the positive direction for all the capabilities except submissive, lazy, and introverted. In fact, 9 of the 16 capabilities show significant positive correlations. A similar pattern was observed for ego resiliency but only two correlations reached significance. Finally, correlations with the Manifest Anxiety Scale were a mixture of positive and negative.

The corresponding pattern of correlations with trait measures (see Table 4) differs in several respects from the pattern with capability measures. As in the previous circumplex literature, several of the traits (lazy, submissive, introverted, cold) exhibit negative correlations with self-esteem, ego resiliency, or interpersonal control. Capabilities show positive or, at worst, nonsignificant correlations with these adjustment measures. Similarly, traits are more highly correlated with anxiety than are capabilities. Apparently, capabilities for antisocial and passive behavior do not detract from adjustment or perceived control; they are simply unrelated. Finally, several traits, but no capabilities, show significant correlations with socially desirable responding.

\section{Discussion}

The capability measures were found to be related to a variety of other indicators of interpersonal effectiveness. As expected, many of the interpersonal capabilities from the BIC were positively correlated with the Interpersonal Control scale from the Spheres of Control battery (Paulhus, 1983). The pattern of correlations indicates that high levels of interpersonal control are associated with the more nurturant (e.g., warm) and the more active (e.g., ambitious) range of capabilities. In fact, the correlations were high: .48 and above for five of the capabilities (warm, ambitious, dominant, gregarious, extraverted). Several of the more negative types of capabilities (e.g., calculating, quarrelsome) were also found to be positively correlated, albeit more weakly, with interpersonal control.

In contrast, Machiavellianism was positively associated with the negative types of capabilities but unrelated to the more positive types. This pattern of correlations was predicted on the basis of the underhanded types of tactics used by individuals high in Machiavellianism (high Machs; Christie \& Geis, 1970). Note that both of the control-oriented scales (Mach IV and the Interpersonal Control scale) correlated positively with the capabilities of being ambitious and calculating. This suggests that although high Machs and those high in interpersonal control tend to use different tactics, both types of individuals can be ambitious and calculating in their attempts to control others.

Overall, our results indicate that the capability measures show an encouraging degree of convergent validity and can be distinguished from socially desirable responding. Further research should explore the relation of the capability measures with other standard measures. Our results also suggest that capability measures can be used to provide a rich description of the kinds of capabilities exhibited by individuals classified according to trait measures. 
Table 4

Personality Correlates of Interpersonal Traits

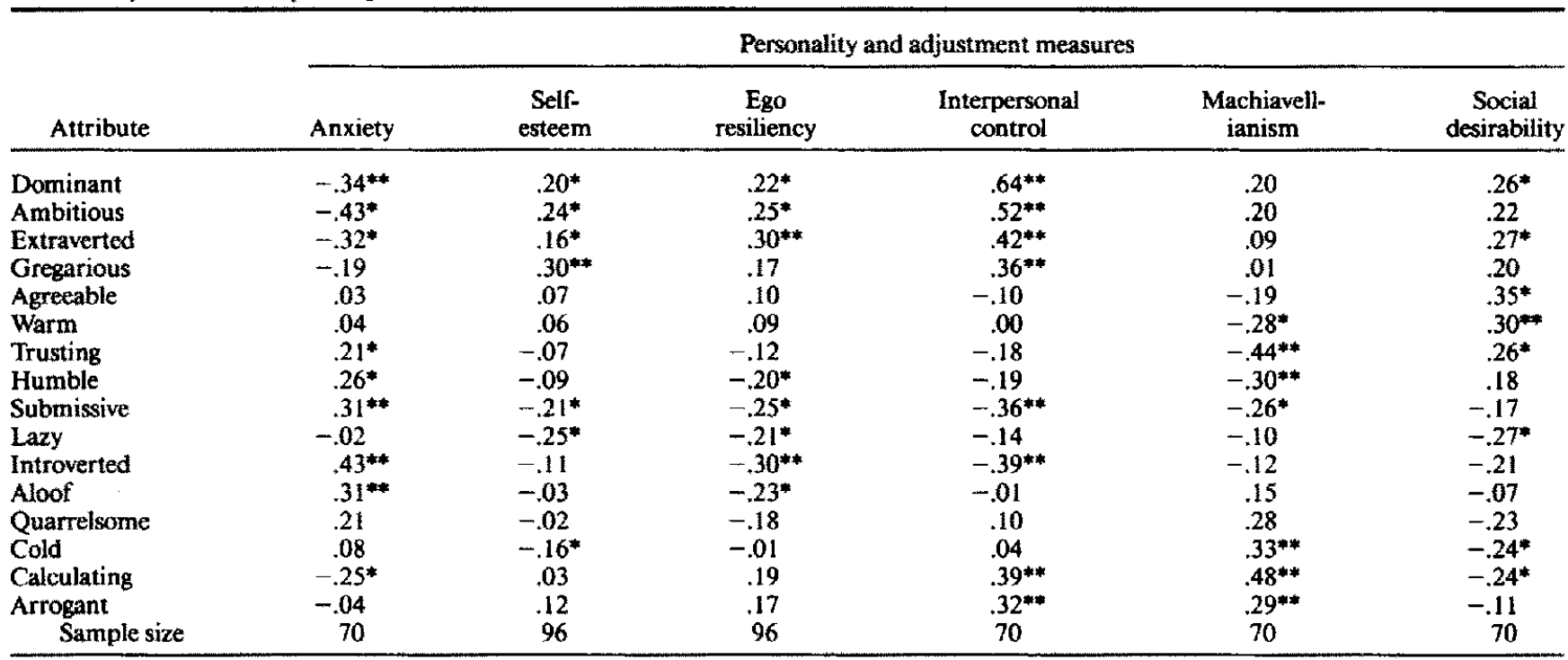

Note. The sample sizes vary across measures because varying sizes of undergraduate classes completed different measures. Anxiety was assessed with Taylor's Manifest Anxiety Scale, self-esteem with Rosenberg's Self-Esteem Scale, ego resiliency with Block's Ego-Resiliency Scale, interpersonal control with Paulhus's Interpersonal Control scale, Machiavellianism with Christie's Mach IV scale, and social desirability with the MarloweCrowne Social Desirability Scale.

* $p<.05$, two-tailed.

$* * p<.01$, two-tailed.

The pattern of correlations between the BIC and the three adjustment measures (anxiety, self-esteem, ego resiliency) was generally consistent with Leary's (1957) predictions. Virtually all the significant correlations indicated positive links between capabilities and adjustment. High self-esteem was linked to 9 of the 16 capabilities. Ego resiliency was associated with the capabilities of being ambitious and extraverted. Low anxiety scores were associated with dominant, ambitious, and extraverted capabilities, suggesting that individuals who are confident of their capabilities in these domains do not experience much general anxiety. Although the causal sequence is not clear, all these results indicate that good adjustment is associated with perceptions of interpersonal capability. In contrast to the capability measures, traits showed more complex correlations with adjustment; for instance, just as many traits were negatively associated as were positively associated with adjustment.

In recent reports (Martin \& Paulhus, 1984; Paulhus \& Martin, 1985), we have used the BIC to explore Leary's proposition that interpersonally flexible people have high self-esteem. After defining flexible individuals as those who report many interpersonal capabilities, we found the predicted association between flexibility and self-esteem. The present results suggest that not all capabilities contribute equally to high self-esteem. Those that seemed to contribute the most were the capabilities associated with traits that were positive and active (dominant, ambitious, extraverted, gregarious) and negative (calculating, arrogant). Also contributing were several other positive capabilities (warm, trusting, agreeable).

In contrast, those capabilities that were both negative and passive (lazy, aloof) did not contribute to high self-esteem. These results are consistent with the finding that passive traits are located at the origin of the capability space (see Figure 3 ). That is, capabilities related to passivity seem to reflect a lack of capabilities for other behaviors.

\section{General Discussion}

Our goal in this article was to elaborate a new conception of personality derived from Wallace's notion of personality abilities. This new conception required a distinction between abilities, capabilities, and traits. In the two studies presented here, capabilities were assessed by asking respondents to estimate the likelihood that they could enact a specific social response if the situation called for it. ${ }^{8}$ In Study 1, respondents reported that they were capable of more interpersonal responses, both positive and negative, than they typically displayed. As predicted, situational anxiety appeared to play a major role in response capabilities. Responses that were given low capability ratings were also associated with high levels of situational anxiety and a tendency to avoid situations requiring such a response.

These results support our contention that the capability is a more valuable unit of analysis than the ability. The self-reported likelihood of demonstrating a specific social response is inseparable from the perception of discomfort. Thus, a capability might aptly be viewed as a perceived ability or performance expectancy.

The structural analyses in Study 1 demonstrated that trait

\footnotetext{
In our most recent work, similar results were obtained by using a more direct phrase: "How capable are you of being dominant when the situation calls for it?"
} 
and capability measures fall on different factors. This factor separation confirms that capability measures are tapping a construct distinct from standard trait measures. It is not known, however, how similar capabilities are to maximal traits as assessed by the format "How dominant can you be?" (Wallace, 1966; Willerman et al., 1976). Work is now underway to address this question.

Study $I$ also revealed that the underlying structure of these capabilities differs dramatically from the well-replicated circumplex structure of the more traditional trait ratings. The capability structure provides a more sanguine picture of personality dynamics: One capability is not constrained by other capabilities. Thus the capability of being cold and hostile does not necessarily preclude being warm and agreeable when it is functional to do so.

\section{Interpersonal Self-Efficacy}

Bandura (1982) defined self-reports of self-efficacy as "judgments of how well one can execute courses of action required to deal with prospective situations" (p. 122). These judgments are said to influence thought parterns, actions, and emotional arousal. This definition sounds remarkably like the self-reported capability described earlier. However, Bandura's theory and the supporting research have focused on the benefits of raising self-efficacy in individuals whose levels are so low as to be dysfunctional. For instance, the self-efficacy of snake phobics has been enhanced by enactive attainment, vicarious experience, verbal persuasion, and anxiety reduction (p. 127). All of these methods effected behavioral change (i.e., improved the ability to approach snakes).

Recently, Moe and Zeiss (1982) have applied self-efficacy theory to the interpersonal realm. They developed measures of selfefficacy for 12 specific social skills. Unlike capabilities, their assessments are based on maximal effort: for example, "Making your very best effort in a conversation, can you be friendly?" As predicted, social skill self-efficacy was associated with low social anxiety. Contrary to predictions, little relation was found with depression.

Although self-efficacy research has focused on dysfunctional deficits, the theory may well apply to the normal range of personality. That is, individual differences in the nondysfunctional range may be driven by the same fundamental dynamics. For example, in the interpersonal realm, we might examine the extent to which individuals perceive themselves to be efficacious in their attempts to be dominant, submissive, nurturant, hostile, and so furth. According to Bandura, these judgments of sels-efficacy would then influence both the degree to which the individual experienced anxiety and how well the behavior would be executed.

The theory of capabilities outlined earlier resembles a theory of interpersonal self-efficasy in the normal range of personality. Self-reports of capabilities can be considered assessments of perceived self-efficacy in the interpersonal realm. Like Bandura's theory of self-efficacy, our theory of capahilities holds that perceptions of self-efficacy can affect situational ankiety. Moreover, the anxiety associated with performing the behavior in a given situation influences perceived self-efficacy. Thus assessments will typically show a strong correlation between selfefficacy and anxiety. In this article, however, these two causal sequences are not distinguished. In sum, we believe that our structural analyses apply equally to capabilities and self-efficacy.

Note that, in both theories, the perceived capability is central. Thus, the self-report is the assessment mode of choice. The subjective nature of the measure is particularly critical in the interpersonal context. Interpersonal capabilities should be even more closely linked with anxiety than should the kinds of capabilities addressed by Bandura. The reason is that social responses require a more complex and sustained engagement than, say, physically approaching a snake. Anxiety can more directly interfere with the interpersonal performance. Thus anticipated anxiety is intrinsic to the self-report of interpersonal capabilities.

Although the concept of a personality capability is closely related to Bandura's conception of self-efficacy, they differ in several respects. We agree with Bandura on the causal relations among perceived self-efficacy, anxiety, and performance. Our emphasis is on interpersonal behavior in the normal range; Bandura's focus is on dysfunctional reactions to phobic stimuli. Moreover, our general approach grew out of a different tradition, namely the work on personality abilities by Wallace, Willerman, and Turner.

\section{Beyond the BIC}

The correlations observed in Study 2 provide some degree of convergent and discriminant validity for the BIC. However, peer-rating and behavioral studies are required to support further the validity of self-reported capabilities. The appropriate context for validating a self-reported capability is the role-playing situation. The subject is told the appropriate role (e.g. dominant, nurturant) and is led to believe be or she is interacting with another subject. This context differs from that used to validate (a) maximal measures where the subject is told to "be as dominant as possible" and (b) trait measures where the subject is given no instructions on how to hehave.

With such validation, the BIC will permit the assessment of a full range of behavioral capabilities in the interpersonal realm. ln Bandura's terms, it proujdes a profile of interpersonal selfefficacy. Given the central role of interpersonal behavior in psychoparhalogy, the BIC constitutes a useful assessment tool for clinical as well as normal samples. Although the BIC was designed for the interpersonal domain, questionnaires with similar question formats could easily be designed to assess capabilities in other domains. For instance, an instrument assessing a full range of phobic responses would be useful. Such an inventory would aid in the search for meaningful clusterings of the various phohias.

\section{Potential Applications}

In addition to providing a new conceptual approach to personality, the capability approach may have a number of applications in exploring other issues in personality. In this article we have illustrated one application: It is possible to elucidate olher personality constructs by detailing the kinds of capabilities associated with those constructs. For example, researchers could provide a richer description of shy indjiduals by determining their precise patters of capabilities. 
A second application is to the examination of certain meaningful patterns of capabilities. For example, the individual capable of more interpersonal responses is thereby more flexible and can adapt to a wide range of situations. Indeed, the total number of capabilities reported on the BIC has been used successfully as an index of an individual's interpersonal flexibility (Paulhus \& Martin, 1985). Unlike current measures that are held to assess interpersonal flexibility (e.g., Bem's, 1974, Bem Sex-Role Inventory; Gough's, 1957, Flexibility scale; Snyder's, 1974, Self-Monitoring scale), the BIC meets two important criteria for measuring interpersonal flexibility: It taps the tailoring of behavior to meet situational demands and it covers the full range of interpersonal behavior. More specific combinations of capabilities and their correlates may also be usefully examined. Recall our finding that having one capability does not preclude having any others. Thus there are many individuals who report a capability for nurturance as well as for hostility. This combination of capabilities may be the basis for a reconception of androgyny (Martin \& Van Oeveren, 1986).

The capability perspective may also be useful for clarifying what respondents mean when they endorse a specific trait. We suspect that many of the personality attributes measured as traits are conceived by respondents as being more like capabilities. Bravery, for example, affords few opportunities and is attributed not on the basis of typicality or frequency, but often on a single event. This possibility provokes a related question: What is the difference between someone with both Trait $\mathrm{X}$ and Capability $\mathrm{X}$ versus someone with only Capability X? Both individuals may be likely to behave in a dominant manner in situations requiring dominance. They may differ only in that the individual with the trait is also more likely to behave in a dominant manner in situations not befitting dominance. Thus people may be more likely to consider their behavior to be traitlike when it tends to occur regardless of the situational demands. These speculations suggest the potential of the capability approach for elucidating the significance of trait attributions.

Finally, the capability concept bears on the issue of personality consistency. Consider, for example, a capability assessment of all 16 interpersonal behaviors in all 16 appropriate situations (a total of 256 pairings). The ratings would indicate, for instance, the likelihood of the person behaving dominantly in situations requiring dominance as well as in inappropriate situations (those requiring nurturance, submissiveness, etc.). From this study we know that individuals differ in the reported likelihood of being dominant in dominance-appropriate situations. Individuals may also differ in the likelihood of being dominant in dominance-inappropriate situations. Let us call the former a functional capability and the latter a dysfunctional capability. Given this distinction, we may determine whether individuals differ in the range of situations for which a particular behavior is exhibited. One person may be capable of dominance across a wide range of situations, whereas others may be capable only in a narrow range of situations. The individual with a single high probability response applied across a large domain of situations would be more likely to show cross-situational consistency, that is, rigidity, in behavior. From a capability perspective, cross-situational consistency in behavior involves both functional and dysfunctional capabilities.

\section{Conclusion}

The interpersonal capability approach provides a new framework for exploring personality. The proposed approach goes be- yond earlier ability conceptions by emphasizing (a) the distinction between abilities and capabilities and (b) the situational appropriateness of behavior. In sum, the approach provides an explicit theoretical and assessment system for adopting the longneglected ability perspective on personality.

\section{References}

Arkowitz, H., Lichenstein, B., McGovern, K., \& Hines, P. (1975). The behavioral assessment of social competence in males. Behavior Therapy, 6, 3-13.

Bandura, A. (1982). Self-efficacy mechanism in human agency. American Psychologist, 37, 122-147.

Bem, S. L. (1974). The measurement of psychological androgyny. Journal of Consulting and Clinical Psychology, 42, 155-162.

Block, J. (1965). The challenge of response sets. New York: AppletonCentury-Crofts.

Broughton, R., \& Paulhus, D. L. (1984, June). Maximal versus typical measures of interpersonal traits. Paper presented at the annual meeting of the Canadian Psychological Association, Ottawa, Ontario, Canada.

Burisch, M. (1984). Approaches to personality inventory construction: A comparison of merits. American Psychologist, 39, 214-227.

Buss, A. H., \& Plomin, R. (1975). A temperament theory of personality development. New York: Wiley.

Buss, D. M., \& Craik, K. H. (1980). The frequency concept of disposition: Dominance and prototypically dominant acts. Journal of Personality, 48, 379-392.

Campbell, D. T., \& Fiske, D. W. (1959). Convergent and discriminant validation by the multitrait-multimethod matrix. Psychological Bulletin, 56, 81-105.

Cattell, R. B. (1957). Personality and motivation: Structure and measurement. Yonkers, NY: World Book.

Christie, R., \& Geis, F. L. (1970). Studies in Machiavellianism. New York: Academic Press.

Crowne, D., \& Marlowe, D. (1960). A new scale of social desirability independent of psychopathology. Journal of Consulting Psychology, 24, 349-354.

Edwards, A. L. (1957). The social desirability variable in personality assessment and research. New York: Dryden.

Eysenck, H. J., \& Eysenck, S. B. G. (1969). Personality structure and measurement. London: Routledge \& Kegan Paul.

Fishbein, M., \& Ajzen, I. (1975). Belief, attitude, intention and behavior: An introduction to theory and research. Reading, MA: AddisonWesley.

Fiske, D. W. (197I). Measuring the concepts of personality. Chicago: Aldine.

Fiske, D. W., \& Butler, J. M. (1963). The experimental conditions for measuring individual differences. Educational and Psychological Measurement, 23, 249-266.

Foa, U. G., \& Foa, E. B. (1974). Societal structures of the mind. Springfield, IL: Charles C. Thomas.

Goldberg, L. R. (1981). Unconfounding situational attributions from uncertain, neutral, and ambiguous ones: A psychometric analysis of descriptions of oneself and various types of others. Journal of Personality and Social Psychology, 41, 517-552.

Gormly, J. (1983). Predicting behavior from personality trait scores. Personality and Social Psychology Bulletin, 9, 267-270.

Gough, H. G. (1957). Manual for the California Psychological Inventory. Palo Alto, CA: Consulting Psychologists Press.

Hersen, M., \& Eisler, R. M. (1976). Social skills training. In W. E. Craighead, A. E. Kazdin, \& M. J. Mahoney (Eds.), Behavior modification: Principles, issues and applications (pp. 361-375). Boston: Houghton Mifflin. 
Hirschberg, N. (1978). A correct treatment of traits. In H. London (Ed.), Personality: A new look at metatheories (pp. 45-68). Washington, DC: Hemisphere.

Klein, H. M., \& Willerman, L. (1979). Psychological masculinity and femininity and typical and maximal dominance expression in women. Journal of Personality and Social Psychology, 37, 20592070.

Klesges, R. C., \& MoGinley, H. (1983). Typical vs. maximal measures of personological variables. Journal of Personality Assessment, 47, 640-647.

Leary, T. (1957). Interpersonal diagnosis of personality. New York: Ronald Press.

Lewinsohn, P. M., Weinstein, M. S., \& Alper, T. (1970). A behavioral approach to the group treatment of depressed persons: A methodological contribution. Journal of Clinical Psychology, 26, 525-532.

Loehlin, J. C., \& Nichols, R. C. (1976). Heredity, environment and personality: A study of 850 twins. Austin: University of Texas Press.

Martin, C. L., \& Paulhus, D. b. (1984, August). A new approach to assessing interpersonal flexibility: Functional flexibility. Paper presented at the annual convention of the American Psychological Association, Toronto.

Martin, C. L., \& Van Oeveren, M. (1986). Reconceiving androgyny in terms of capabilities. Unpublished manuscript, University of British Columbia, Vancouver, Canada.

McClelland, D. C. (1951). Personality. New York: Dryden-Sloan.

Megargee, E. I. (1966). Undercontrolled and overcontrolled personality types in extreme antisocial aggression. Psychological Monographs, 80 (3, Whole No. 611).

Moe, K. O., \& Zeiss, A. M. (1982). Measuring self-efficacy expectations for social skills: A methodological inquiry. Cognitive Therapy and Research, 6, 191-205.

Nietzel, M. T., \& Bernstein, D. A. (1976). Effects of instructionally mediated demands on the behavioral assessment of assertiveness. Journal of Consulting and Clinical Psychology, 44, 500.

Paulhus, D. L. (1983). Sphere-specific measures of perceived control. Journal of Personality and Social Psychology, 44, 1253-1265.

Paulhus, D. L. (1984). Two-component models of socially desirable responding. Journal of Personality and Social Psychology, 46, 598-609.

Paulhus, D. L., \& Martin, C. L. (1985). Interpersonal functional flexibility: $A$ new method for assessing interpersonal flexibility. Manuscript submitted for publication.

Paulhus, D. L., \& Martin, C. L. (1986). Predicting adult temperament from minor physical anomalies. Journal of Personality and Social Psychology, 50, 1235-1239.

Percell, L. P., Berwick, P. T., \& Beigel, A. (1974). The effects of assertiveness training on self-concept and anxiety. Archives of General Psychiatry, 31, 502-504.

Rosenberg, M. (1965). Society and the adolescent self-image. Princeton, NJ: Princeton University Press.
Russell, J. A. (1980). A circumplex model of affect. Journal of Personality and Social Psychology, 39, 1161-1178.

Ryle, G. (1949). The concept of mind. New York: Barnes \& Noble.

Shields, J. (1976). Heredity and environment. In H. J. Eysenck \& G. D. Wilson (Eds.), A textbook of human psychology (pp. 145-160). Baltimore, MD: University Park Press.

Smith, P. M. (1984). Motivational styles in intergroup bargaining. Manuscript submitted for publication.

Snyder, M. (1974). Self-monitoring of expressive behavior. Journal of Personality and Social Psychology, 30, 526-537.

Sullivan, H. S. (1953). The interpersonal theory of psychiatry. New York: Norton.

Taylor, J. (1953). A personality scale of manifest anxiety. Journal of $A b$ normal and Social Psychology, 48, 285-290.

Turner, R. G. (1978). Consistency, self-consciousness, and the predictive validity of typical and maximal personality measures. Journal of Research in Personality, 12, 117-132.

Turner, R. G. (1983, August). Social anxiety and performance in ambiguous versus structured situations. Paper presented at the annual convention of the American Psychological Association, Anaheim, CA.

Twentyman, C. T., \& McFall, R. M. (1975). Behavioral training of social skills in shy males. Journal of Consulting and Clinical Psychology, 43, 384-395.

Wallace, J. (1966). An abilities conception of personality: Some implications for personality measurement. American Psychologist, 21, 132-138.

Wallace, J. (1967). What units shall we employ? Allport's question recited. Journal of Consulting Psychology, 31, 56-64.

White, W. C., \& Berger, F. (1976). Behavior rehearsal and social competence: A pilot study. Journal of Consulting Psychology, 23, 567-570.

Wiggins, J. S. (1974, February). In defense of traits. Paper presented at the Ninth Annual Symposium on Recent Developments in the use of the MMPI, Los Angeles.

Wiggins, J. S. (1979). A psychological taxonomy of trait-descriptive terms: The interpersonal domain. Journal of Personality and Social Psychology, 37, 395-412.

Wiggins, J. S. (1980). Circumplex models of interpersonal behavior. In L. Wheeler (Ed.), Review of personality and social psychology (Vol. 1, pp. 265-294). Beverly Hills, CA: Sage.

Wiggins, J. S., \& Broughton, R. (1985). The interpersonal circle: A structural model for the integration of personality research. In R. Hogan \& W. H. Jones (Eds.), Perspectives in personality: Theory measurement and interpersonal dynamics (Vol. 1, pp. 1-47). Greenwich, CT: JAI Press.

Willerman, L., Turner, R. G., \& Peterson, M. (1976). A comparison of the predictive validity of typical and maximal personality measures. Journal of Research in Personality, 10, 482-492.

Wolpe, J. (1969). The practice of behavior therapy. New York: Pergamon Press.

Received May 23, 1985

Revision received December 18, 1985 